\title{
AN ESTIMATE FROM BELOW FOR THE BUFFON NEEDLE PROBABILITY OF THE FOUR-CORNER CANTOR SET
}

\author{
Michael Bateman and Alexander Volberg
}

\begin{abstract}
Let $\mathcal{C}_{n}$ be the $n$-th generation in the construction of the middle-half Cantor set. The Cartesian square $\mathcal{K}_{n}=\mathcal{C}_{n} \times \mathcal{C}_{n}$ consists of $4^{n}$ squares of side-length $4^{-n}$. The chance that a long needle thrown at random in the unit square will meet $\mathcal{K}_{n}$ is essentially the average length of the projections of $\mathcal{K}_{n}$, also known as the Favard length of $\mathcal{K}_{n}$. A classical theorem of Besicovitch implies that the Favard length of $\mathcal{K}_{n}$ tends to zero. It is still an open problem to determine its exact rate of decay. Until recently, the only explicit upper bound was $\exp \left(-c \log _{*} n\right)$, due to Peres and Solomyak. $\left(\log _{*} n\right.$ is the number of times one needs to take log to obtain a number less than 1 starting from $n)$. In [11] the power estimate from above was obtained. The exponent in [11] was less than $1 / 6$ but could have been slightly improved. On the other hand, a simple estimate shows that from below we have the estimate $\frac{c}{n}$. Here we apply the idea from [4], [1] to show that the estimate from below can be in fact improved to $c \frac{\log n}{n}$. This is in drastic contrast to the case of random Cantor sets studied in [13].
\end{abstract}

\section{Introduction}

The four-corner Cantor set $\mathcal{K}$ is constructed by replacing the unit square by four sub-squares of side length $1 / 4$ at its corners, and iterating this operation in a selfsimilar manner in each sub-square. More formally, consider the set $\mathcal{C}_{n}$ that is the union of $2^{n}$ segments:

$$
\mathcal{C}_{n}=\bigcup_{a_{j} \in\{0,3\}, j=1, . ., n}\left[\sum_{j=1}^{n} a_{j} 4^{-j}, \sum_{j=1}^{n} a_{j} 4^{-j}+4^{-n}\right],
$$

and let the middle half Cantor set be

$$
\mathcal{C}:=\bigcap_{n=1}^{\infty} \mathcal{C}_{n}
$$

Received by the editors July 24, 2008.

1991 Mathematics Subject Classification. Primary: 28A80. FractalsSecondary: 28A75, Length, area, volume, other geometric measure theory 60D05, Geometric probability, stochastic geometry, random sets $28 \mathrm{~A} 78$ Hausdorff and packing measures.

Research of the authors was supported in part by NSF grants DMS-0501067 (Volberg) and DMS0653763 (Bateman). 

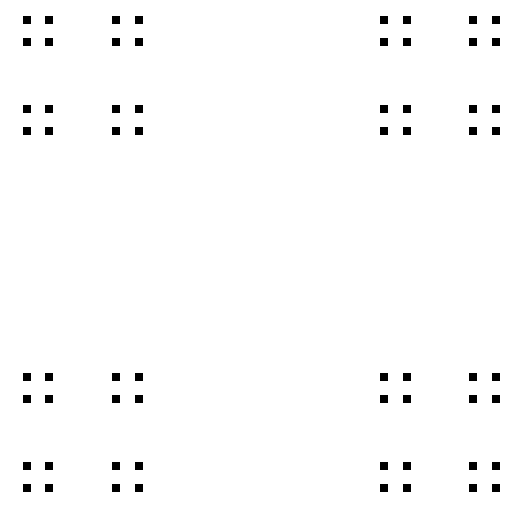

FigurE $1 . \mathcal{K}_{3}$, the third stage of the construction of $\mathcal{K}$.

(It can also be written as $\mathcal{C}=\left\{\sum_{n=1}^{\infty} a_{n} 4^{-n}: a_{n} \in\{0,3\}\right\}$.) The four corner Cantor set $\mathcal{K}$ is the Cartesian square $\mathcal{C} \times \mathcal{C}$.

Since the one-dimensional Hausdorff measure of $\mathcal{K}$ satisfies $0<\mathcal{H}^{1}(\mathcal{K})<\infty$ and the projections of $\mathcal{K}$ in two distinct directions have zero length, a theorem of Besicovitch (see [3, Theorem 6.13]) yields that the projection of $\mathcal{K}$ to almost every line through the origin has zero length. This is equivalent to saying that the Favard length of $\mathcal{K}$ equals zero. Recall (see [2, p. 357]) that the Favard length of a planar set $E$ is defined by

$$
\operatorname{Fav}(E)=\frac{1}{\pi} \int_{0}^{\pi}\left|\operatorname{Proj} \mathcal{R}_{\theta} E\right| d \theta,
$$

where Proj denotes the orthogonal projection from $\mathbb{R}^{2}$ to the horizontal axis, $\mathcal{R}_{\theta}$ is the counterclockwise rotation by angle $\theta$, and $|A|$ denotes the Lebesgue measure of a measurable set $A \subset \mathbb{R}$. The Favard length of a set $E$ in the unit square has a probabilistic interpretation: up to a constant factor, it is the probability that the "Buffon's needle," a long line segment dropped at random, hits E. (More precisely, suppose the needle's length is infinite, pick its direction uniformly at random, and then locate the needle in a uniformly chosen position in that direction, at distance at most $\sqrt{2}$ from the center of the unit square.)

The set $\mathcal{K}_{n}=\mathcal{C}_{n}^{2}$ is a union of $4^{n}$ squares with side length $4^{-n}$ (see Figure 1 for a picture of $\left.\mathcal{K}_{3}\right)$. By the dominated convergence theorem, $\operatorname{Fav}(\mathcal{K})=0$ implies $\lim _{n \rightarrow \infty} \operatorname{Fav}\left(\mathcal{K}_{n}\right)=0$. We are interested in good estimates for $\operatorname{Fav}\left(\mathcal{K}_{n}\right)$ as $n \rightarrow \infty$. A lower bound $\operatorname{Fav}\left(\mathcal{K}_{n}\right) \geq \frac{c}{n}$ for some $c>0$ follows from Mattila [8, 1.4]. Peres and Solomyak [13] proved that

$$
\operatorname{Fav}\left(\mathcal{K}_{n}\right) \leq C \exp \left[-a \log _{*} n\right] \quad \text { for all } n \in \mathbb{N}
$$


where

$$
\log _{*} n=\min \{k \geq 0: \underbrace{\log \log \ldots \log n}_{k} n \leq 1\} .
$$

This result can be viewed as an attempt to make a quantitative statement out of a qualitative Besicovitch projection theorem [2], [15], using this canonical example of a Besicovitch irregular set. It is very interesting to consider quantitative analogs of Besicovitch theorem in general. The reader can find more of that in [15].

In [11] the following estimate from above was obtained

$$
\operatorname{Fav}\left(\mathcal{K}_{n}\right) \leq \frac{C_{\tau}}{n^{\tau}},
$$

where $\tau$ was strictly less than $1 / 6$. This can be slightly improved, but it is still a long way till $\tau=1$. Here we show, using the idea of [1], [4], that $\tau=1$ is impossible.

Theorem 1. There exists $c>0$ such that

$$
\operatorname{Fav}\left(\mathcal{K}_{n}\right) \geq c \frac{\log n}{n} \quad \text { for all } n \in \mathbb{N} .
$$

Remark. This result is somewhat surprising in light of the probabilistic result in [13]. There, the authors consider planar Cantor sets constructed randomly as follows. Starting from the unit square $U$, divide $U$ into four equal squares $U_{1}, U_{2}, U_{3}, U_{4}$. Similarly divide each of these into four squares $U_{j 1}, U_{j 2}, U_{j 3}, U_{j 4}$. For each $j$, randomly choose one square $U_{j k}$ (of side length $\frac{1}{16}$ ). The four chosen squares form the first level $\tilde{\mathcal{K}}_{1}$. Repeat this process, always choosing the next generation randomly. The authors in [13] show that one expects

$$
\frac{1}{C n} \leq \operatorname{Fav}\left(\tilde{\mathcal{K}}_{n}\right) \leq \frac{C}{n} .
$$

Proof of Theorem 1. The proof is an immediate corollary of the idea of [4] if one applies the duality between Cantor sets and Kakeya sets from [9]. As a "warm-up" we are going to prove a much simpler estimate

$$
\operatorname{Fav}\left(\mathcal{K}_{n}\right) \geq \frac{c}{n} \quad \text { for all } n \in \mathbb{N} .
$$

This does not require [1], [4].

In what follows the square means only the Cantor square. Let $L_{\theta}$ be the line passing through the origin at an angle $\theta$ with the $x$-axis. Let $f_{n, \theta}(x)$ denote the number of squares in $\mathcal{K}_{n}$ whose orthogonal projection onto the line $L_{\theta}$ contains a point $x$ of this line. For each square $Q$ contained in $\mathcal{K}_{n}$ with sidelength $4^{-n}$, let $\chi_{Q, \theta}$ be the characteristic function of the projection of $Q$ onto $L_{\theta}$. Let $\ell(Q)$ be the sidelength of a square $Q$. Then $f_{n, \theta}(x)=\sum_{Q, \ell(Q)=4^{-n}} \chi_{Q, \theta}(x)$. Therefore,

$$
\iint f_{n, \theta}(x) d x d \theta \asymp 4^{n} \cdot 4^{-n}=1 .
$$

Let us denote the support of $f_{n, \theta}(x)$ by $E_{n, \theta},\left|E_{n, \theta}\right|$ being its one-dimensional measure on $L_{\theta}$. 
Knowing the first and second moment of $f_{n, \theta}(x)$ we can estimate $\int\left|E_{n, \theta}\right| d \theta$ by using Cauchy inequality twice:

Hence,

$$
\begin{gathered}
1 \asymp \iint f_{n, \theta} d x d \theta \leq \int\left|E_{n, \theta}\right|^{\frac{1}{2}}\left(\int f_{n, \theta}^{2}(x) d x\right)^{\frac{1}{2}} d \theta \leq \\
\left(\int\left|E_{n, \theta}\right| d \theta\right)^{\frac{1}{2}}\left(\iint f_{n, \theta}^{2}(x) d x d \theta\right)^{\frac{1}{2}} .
\end{gathered}
$$

$$
\int\left|E_{n, \theta}\right| d \theta \geq c \frac{1}{\iint f_{n, \theta}^{2}(x) d x d \theta} .
$$

Now

$$
\iint f_{n, \theta}^{2}(x) d x d \theta=\sum_{Q, Q^{\prime}, \ell(Q)=\ell\left(Q^{\prime}\right)=4^{-n}} \iint \chi_{Q, \theta}(x) \chi_{Q^{\prime}, \theta}(x) d x d \theta .
$$

So for each pair $P=\left(Q, Q^{\prime}\right), \ell(Q)=\ell\left(Q^{\prime}\right)=4^{-n}\left(Q\right.$ and $Q^{\prime}$ may coincide) we consider

$$
p_{P}:=\int\left|\operatorname{Proj}_{\theta} Q \cap \operatorname{Proj}_{\theta} Q^{\prime}\right| d \theta .
$$

We define a distance-type function on pairs of squares. For $k=0,1, \ldots, n$, we call a pair $P=\left(Q, Q^{\prime}\right)$ a $k$-pair if $Q, Q^{\prime}$ are in a square of sidelength $4^{-k}$, but not in any square of sidelength $4^{-k-1}$. We have $4^{k}$ of $4^{-k}$-squares, so we have $\asymp 4^{k} \cdot\left(4^{n-k}\right)^{2}$ different $k$-pairs. For each $k$-pair $P$ we obviously have

$$
p_{P} \leq C 4^{-n} 4^{k-n} \text {. }
$$

Putting this together we get

$$
\begin{gathered}
\iint f_{n, \theta}^{2}(x) d x d \theta=\sum_{P} p_{P}=\sum_{k=0}^{n} \sum_{P \text { is a } k-\text { pair }} p_{P} \\
\leq C \sum_{k=0}^{n-1} \sum_{P \text { is a } k \text {-pair }} 4^{-n} 4^{k-n} \leq C \sum_{k=0}^{n-1} 4^{k} \cdot\left(4^{n-k}\right)^{2} 4^{-n} 4^{k-n} \leq C n .
\end{gathered}
$$

This estimate and (1.5) give us

$$
\int\left|E_{n, \theta}\right| d \theta \geq \frac{c}{n}
$$

To prove (1.2) one needs to count pairs in a much more interesting way, which one gets from [1].

First we consider axis $0 X$, where 0 is the origin and the axis has angle $\arctan \frac{1}{2}$ with the horizontal axis. We also need $0 Y$, the orthogonal axis. Project the original unit square onto $0 X$. We obtain the segment $I_{0}:=[0, L], L=\sqrt{2} \cos \left(\frac{\pi}{4}-\arctan \frac{1}{2}\right)$ on $0 X$. Notice that projections of Cantor squares of size $4^{-k}, k=0, \ldots, n$, generate the 4 -adic grid on $I_{0}=[0, L]$. Intervals of this 4 -adic grid will be called $I_{\sigma}$, where $\sigma$ is the word of length at most $n$ in the alphabet of $\{0,1,2,3\}$.

We have $4^{n}$ points that are the projections of the centers of $4^{n}$ squares $Q$ of size $4^{-n}$. We will call this set $S$, and use the notation $s$ (maybe with indices) for elements of $S$. Because of our choice of axis $0 X$, this correspondence between elements of $S$ 
and squares of sidelength $4^{-n}$ is one-to-one. Let $y_{s}$ be the $0 Y$ coordinate of the center of $Q_{s}$. Note that each $s$ is the center of an interval $I_{\sigma}$, and that the projections of all cubes $Q$ onto this axis are disjoint. This is an important feature of the argument.

Along with the usual Euclidean distance $\left|s_{1}-s_{2}\right|$ between the points $s_{1}, s_{2} \in S$, we have another very simple distance which will play a crucial role in proving (1.2). Namely,

$$
d\left(s_{1}, s_{2}\right):=\min \left\{\left|I_{\sigma}\right|, s_{1} \in I_{\sigma} s_{2} \in I_{\sigma}\right\} .
$$

This is just the usual 4 -adic distance scaled by $L$. Of course $\left|s_{1}-s_{2}\right| \leq d\left(s_{1}, s_{2}\right)$.

For $j=0,1, . ., \log n, k \in[-n+j, 0]$, we call pair $P$ a $(j, k)$-pair , if

$$
\frac{\left|s_{1}-s_{2}\right|}{\left|y_{s_{1}}-y_{s_{2}}\right|} \asymp 4^{-j},\left|s_{1}-s_{2}\right| \asymp 4^{-k-j} .
$$

Now the pair $P=\left(Q, Q^{\prime}\right)$ of squares of size $4^{-n}$ is just a pair $\left(s_{1}, s_{2}\right), s_{i} \in S$.

For every $(j, k)$-pair $P=\left(s_{1}, s_{2}\right)$ one immediately has

$$
p_{P} \leq C \frac{1}{4^{n}} \cdot \frac{4^{-n}}{\left|y_{s_{1}}-y_{s_{2}}\right|} .
$$

where $p_{P}$ is as in (1.6). Now we want to estimate the number $A_{j, k}$ of all $(j, k)$-pairs. If $\left(s_{1}, s_{2}\right)$ is a $(j, k)$-pair, then

$$
\left|s_{1}-s_{2}\right| \asymp 4^{-k-j}
$$

But also

$$
4^{j}\left|s_{1}-s_{2}\right| \leq C\left|y_{s_{1}}-y_{s_{2}}\right|,
$$

and

$$
\left|y_{s_{1}}-y_{s_{2}}\right| \leq C^{\prime} d\left(s_{1}, s_{2}\right) .
$$

The last inequality is obvious but it is the most crucial for the proof!

This is because we just obtained $d\left(s_{1}, s_{2}\right) \geq c 4^{-k}$. How many 4 -adic intervals are such that $d\left(s_{1}, s_{2}\right) \geq c 4^{-k} \geq 4^{-k-a}$ ( $a$ is absolute), and $\left|s_{1}-s_{2}\right| \asymp 4^{-k-j}$ ? Corresponding two 4 -adic intervals of size $4^{-n}$ should be both in $C 4^{-k-j}$-neighborhood of the 4 -adic points of $1,2,3, \ldots, k, k+1, . ., k+a$-generations. We have $4,4^{2}, \ldots, 4^{k+a}$ such points correspondingly.

Therefore,

$$
A_{j, k} \leq C \sum_{m=0}^{k+a} 4^{m}\left(\frac{4^{-k-j}}{4^{-n}}\right)^{2}=C 4^{2 n-k-2 j}
$$

Another way to count the number of $(j, k)$ pairs is as follows.

Recall that we have a 4 -adic structure on the axis $0 X$, and that each $s_{j}$ is the center of an interval of length $4^{-n} L$. Hence we may identify each $s_{j}$ with an $n$-digit string of numbers in $\{0,1,2,3\}$. Say $s_{1}=a_{1} a_{2} \ldots a_{n}$ and $s_{2}=b_{1} b_{2} \ldots b_{n}$. If $\left(s_{1}, s_{2}\right)$ is a $(j, k)$ pair, then there is some $i \in\{0,1, \ldots, k+a\}$ such that $d\left(s_{1}, s_{2}\right)=L 4^{-k-a+i}$. Further, we have that $\left|s_{1}-s_{2}\right| \leq L 4^{-k-j}$. Hence we know that $a_{1}=b_{1}, a_{2}=b_{2}, \ldots$, $a_{k+a-i}=b_{k+a-i}$, because $d\left(s_{1}, s_{2}\right)=L 4^{-k-a+i}$, and we know that the next $j-a+i$ digits of both $a$ and $b$ are almost uniquely determined, because $\left|s_{1}-s_{2}\right| \leq L 4^{-k-j}$. I.e., we must have either 


$$
a=a_{1} a_{2} \ldots a_{k+a-i} 033 \ldots 33 a_{k+j} \ldots a_{n} \text { and } b=a_{1} a_{2} \ldots a_{k+a} 100 \ldots 00 b_{k+j} \ldots b_{n}
$$

or

$$
a=a_{1} a_{2} \ldots a_{k+a-i} 133 \ldots 33 a_{k+j} \ldots a_{n} \text { and } b=a_{1} a_{2} \ldots a_{k+a} 200 \ldots 00 b_{k+j} \ldots b_{n}
$$

or

$$
a=a_{1} a_{2} \ldots a_{k+a-i} 233 \ldots 33 a_{k+j} \ldots a_{n} \text { and } b=a_{1} a_{2} \ldots a_{k+a} 300 \ldots 00 b_{k+j} \ldots b_{n},
$$

(possibly with the roles of $a$ and $b$ switched). Hence for each $i=0,1, \ldots, k+a$, we have that the number of $(j, k)$ pairs $\left(s_{1}, s_{2}\right)$ with $d\left(s_{1}, s_{2}\right)=L 4^{-k-a+i}$ is less than $C 4^{k+a-i} 4^{2(n-k-j)}=C 4^{2 n-k-2 j-i}$. Hence

$$
A_{j, k} \leq C \sum_{i=0}^{k+a} 4^{2 n-k-2 j-i} \leq C 4^{2 n-k-2 j} .
$$

Using this and (1.7) we get

$$
\sum_{p \in(j, k)-\text { pairs }} p_{P} \leq C 4^{2 n-k-2 j} \frac{4^{-2 n}}{4^{-k}} \asymp 4^{-2 j} .
$$

The union of all $(j, k)$-pairs over all $k$ is called: $\mathcal{P}_{j}^{\prime}$.

So fix $j$, and get

$$
\sum_{p \in \mathcal{P}_{j}^{\prime}} p_{P}=\sum_{k=-n+j}^{0} \sum_{p \in(j, k)-\text { pairs }} p_{P} \leq C \frac{n}{4^{2 j}} .
$$

Now let $J_{j}:=\left[c_{1} 4^{-j}, c_{2} 4^{-j}\right]$, where $c_{1}$ is sufficiently small and $c_{2}$ is sufficiently large. These are intervals of angles $\theta$ with respect to the axis $0 X$, where zero angle means we are line parallel to the axis $0 X$.

Here is a crucial geometric observation:

$$
\begin{aligned}
& \text { If } P=\left(Q, Q^{\prime}\right), Q \neq Q^{\prime} \\
& \qquad \operatorname{Proj}_{\theta} Q \cap \operatorname{Proj}_{\theta} Q^{\prime} \neq \emptyset, \theta \in J_{j} \text { then } P \in \mathcal{P}_{j}^{\prime} .
\end{aligned}
$$

Let us throw into $\mathcal{P}_{j}^{\prime}$ also all $(Q, Q)$ pairs. The resulting collection is called $j$-pairs: $\mathcal{P}_{j}$. As

$$
\begin{gathered}
\int_{J_{j}}\left|E_{n, \theta}\right| d \theta \geq c \frac{\left(\int_{J_{j}} \int f_{n, \theta} d x d \theta\right)^{2}}{\int_{J_{j}} \int f_{n, \theta}^{2} d x d \theta}, \\
\int_{J_{j}} \int f_{n, \theta}^{2} d x d \theta \leq \sum_{p \in \mathcal{P}_{j}} p_{P} \leq C \frac{n}{4^{2 j}}+ \\
\int_{J_{j}} \int_{P=(Q, Q), \ell(Q)=4^{-n}} \chi_{Q, \theta}(x) d x d \theta \leq C \frac{n}{4^{2 j}}+C 4^{-j} 4^{n} 4^{-n} \leq C \frac{n}{4^{2 j}},
\end{gathered}
$$

and

$$
\int_{J_{j}} \int f_{n, \theta} d x d \theta \geq c\left|J_{j}\right| \cdot 4^{n} \cdot 4^{-n} \asymp 4^{-j},
$$


we combine this to obtain

$$
\int_{J_{j}}\left|E_{n, \theta}\right| d \theta \geq c 4^{-2 j} \frac{4^{2 j}}{n}=\frac{c}{n} .
$$

Remark. Notice that (1.11) stops to be valid if $j>\log _{4} n+$ Const, because the contribution from the $(Q, Q)$ pairs is no longer negligible. This explains why we did not get a better estimate from below than that in the Theorem.

Summing (1.12) over $j=0, \ldots, \log n$ we obtain (1.2). Theorem is completely proved.

\section{Median value of $\left|E_{n, \theta}\right|$}

Question 1. What is the median value of $\left|E_{n, \theta}\right|$ ?

Let us call this median value $M_{n}$. We can prove the following simple theorem, which immediately implies (1.3) of course.

Theorem 2. $M_{n} \geq \frac{c}{n}$.

Proof. We are going to prove

$$
\int \frac{1}{\left|E_{n, \theta}\right|} d \theta \leq C n
$$

If one uses Tchebyshev's inequality this immediately gives $M_{n} \geq \frac{c}{n}$.

To prove (2.1) we use [9]. Let us fix a small positive $\varepsilon$, and let $\mu_{n}$ be an equidistributed measure on $\mathcal{C}_{n}$. Let $\operatorname{Proj}_{\theta}$ stand (as always) for the orthogonal projection onto line $L_{\theta}$. Notice that given two points $z, \zeta \in \mathbb{C}$ we have

$$
\frac{\varepsilon}{|z-\zeta|} \asymp \mid\left\{\theta:\left|\operatorname{Proj}_{\theta}(z)-\operatorname{Proj}_{\theta}(\zeta)\right| \leq \varepsilon \| \mid\right.
$$

Using this we write

$$
\iint \frac{\varepsilon}{|z-\zeta|} d \mu_{n}(z) d \mu_{n}(\zeta) \asymp \iint\left|\left\{\theta:\left|\operatorname{Proj}_{\theta}(z)-\operatorname{Proj}_{\theta}(\zeta)\right| \leq \varepsilon\right\}\right| d \mu_{n}(z) d \mu_{n}(\zeta)
$$

Introduce

$$
\Phi_{\varepsilon}(x)=\left\{\begin{array}{l}
1, \text { if } 0 \leq x \leq \varepsilon \\
0, \text { otherwise }
\end{array}\right.
$$

Then we repeat

$$
\begin{gathered}
\iint \frac{\varepsilon}{|z-\zeta|} d \mu_{n}(z) d \mu_{n}(\zeta) \asymp \iiint \Phi_{\varepsilon}\left(\left|\operatorname{Proj}_{\theta}(z)-\operatorname{Proj}_{\theta}(\zeta)\right|\right) d \mu_{n}(z) d \mu_{n}(\zeta) d \theta= \\
\iiint \Phi_{\varepsilon}(|x-y|) d \mu_{n, \theta}(x) d \mu_{n, \theta}(y) d \theta,
\end{gathered}
$$

where $d \mu_{n, \theta}$ is the projection of the measure $\mu_{n}$ on the line $L_{\theta}$. In our old notation

$$
d \mu_{n, \theta}=f_{n, \theta}(x) d x .
$$

Of course

$$
\int \Phi_{\varepsilon}(|x-y|) d \mu_{n, \theta}(y)=\mu_{n, \theta}(B(x, \varepsilon)),
$$


and finally we get

$$
\iint \frac{1}{|z-\zeta|} d \mu_{n}(z) d \mu_{n}(\zeta) \geq c \iint \frac{\mu_{n, \theta}(B(x, \varepsilon))}{\varepsilon} d \mu_{n, \theta}(x) d \theta .
$$

The left hand side is $\leq C n$. One can see this by noting that for each square $Q$ of side length $4^{-n}$ in $\mathcal{K}_{n}$, and for each $k=0,1, \ldots, n$, there are $4^{n-k}$ squares $Q^{\prime}$ at distance $4^{-k}$.

In (2.3) we now use Fatou's lemma:

$$
\int \liminf _{\varepsilon \rightarrow 0} \frac{\mu_{n, \theta}(B(x, \varepsilon))}{\varepsilon} d \mu_{n, \theta}(x) d \theta \leq C n .
$$

Recalling (2.2) we obtain

$$
\iint_{E_{n, \theta}} f_{n, \theta}(x)^{2} d x d \theta \leq C n .
$$

Recalling (1.4) we can rewrite it as

$$
\int \frac{\int_{E_{n, \theta}} f_{n, \theta}(x)^{2} d x}{\left(\int_{E_{n, \theta}} f_{n, \theta}(x) d x\right)^{2}} d \theta \leq C n .
$$

By Cauchy inequality

$$
\frac{1}{\left|E_{n, \theta}\right|} \leq \frac{\int_{E_{n, \theta}} f_{n, \theta}(x)^{2} d x}{\left(\int_{E_{n, \theta}} f_{n, \theta}(x) d x\right)^{2}} .
$$

Combine this and (2.6) and obtain the desired estimate

$$
\int \frac{1}{\left|E_{n, \theta}\right|} d \theta \leq C n
$$

Inequality (2.1) and, therefore, Theorem 2 are completely proved.

\section{Sierpiński's Cantor set}

Consider now another Cantor set, which, by analogy with Sierpiński's gasket, we call Sierpiński's Cantor set $\mathcal{S}$. We take an equilateral triangle with side lenghth 1 , leave 3 triangles of size $1 / 3$ at each corner, and then continue this for $n$ generations. On step $n$ we get $3^{n}$ equilateral tringles of size $3^{-n}$. Call this union of triangles $\mathcal{S}_{n}$. Its intersection is $\mathcal{S}$,

$$
0<H^{1}(\mathcal{S})<\infty
$$

and this is a Besicovitch irregular set, so, by Besicovitch projection theorem (see [10])

$$
\zeta_{n}:=\int\left|\mathcal{S}_{n, \theta}\right| d \theta \rightarrow 0, n \rightarrow \infty
$$

Question 2. What is the order of magnitude of $\zeta_{n}$ ?

This is the same question, which we had for 4-corner Cantor set.

Absolutely the same reasoning as above proves

Theorem 3.

$$
\zeta_{n}=\int\left|\mathcal{S}_{n, \theta}\right| d \theta \geq c \frac{\log n}{n} .
$$


In fact, projection of the triangles on the base side generate 3 -adic lattice on the base side. Then we notice that (1.8) and (1.10) hold now as well. The proof is the same after these observations.

\section{References}

1. M. Bateman, N.Katz, Kakeya sets in Cantor directions, arXiv:math/0609187v1, 2006, pp. 1-10.

2. A. S. Besicovitch, Tangential properties of sets and arcs of infinite linear measure, Bull. Amer. Math. Soc. 66 (1960), 353-359.

3. K. J. Falconer, The geometry of fractal sets. Cambridge Tracts in Mathematics, 85. C.U.P., Cambridge-New York, (1986).

4. Katz, N. H., A counterexample for maximal operators over a Cantor set of directions Math. Res. Let. 3 (1996), pp. 527-536.

5. U. Keich, On $L^{p}$ bounds for Kakeya maximal functions and the Minkowski dimension in $\mathbb{R}^{2}$, Bull. London. Math. Soc. 31 (1999), pp. 213-221.

6. R. Kenyon, Projecting the one-dimensional Sierpinski gasket, Israel J. Math. 97 (1997), 221238.

7. J. C. Lagarias and Y. Wang, Tiling the line with translates of one tile, Invent. Math.124 (1996), 341-365.

8. P. Mattila, Orthogonal projections, Riesz capacities and Minkowski content, Indiana Univ. Math. J. 39 (1990), 185-198.

9. P. Mattila, Hausdorff dimension, projections, and the Fourier tarnsform, Publ. Mat., 48 (2004), pp. 3-48.

10. P. Mattila, Geometry of Sets and Measures in Euclidean Spaces, Cambridge University Press, 1995.

11. F. Nazarov, Y. Peres, A. Volberg, The power law for the Buffon needle probability of the fourcorner Cantor set, arXiv:0801.2942, 2008, pp. 1-15.

12. Y. Peres, K. Simon and B. Solomyak, Self-similar sets of zero Hausdorff measure and positive packing measure, Israel J. Math. 117 (2000),353-379.

13. Y. Peres and B. Solomyak, How likely is Buffon's needle to fall near a planar Cantor set? Pacific J. Math. 204, 2 (2002), 473-496.

14. I. J. Schoenberg, On the Besicovitch-Perron solution of the Kakeya problem, Studies in mathmatical analysis and related topics,

15. T. Tao, A quantitative version of the Besicovitch projection theorem via multiscale analysis, $\mathrm{pp}$. 1-28, arXiv:0706.2446v1 [math.CA] 18 Jun 2007.

Department of Mathematics, Indiana University, 831 East 3rd St, Bloomington, in 47405

E-mail address: mdbatema@indiana.edu

Current address: Department of Mathematics, UCLA, Box 951555 Los Angeles, CA 90095-1555

E-mail address: bateman@math.ucla.edu

Alexander Volberg, Department of Mathematics, Michigan State University, East Lansing, Mi 48824, and The University of Edinburgh, James Clerk Maxwell Building, The King's Buildings, Mayfield Road, Edinburgh, Scotland EH9 3JZ

E-mail address: volberg@math.msu.edu

E-mail address: a.volberg@ed.ac.uk 